\title{
Resultado perinatal de embarazos gemelares atendidos en dos instituciones de alta complejidad en Bogotá, Colombia
}

\author{
Saulo Molina Giraldo, $M S c^{1}$, José María Araméndiz ${ }^{1}$, Sandra Liliana Beltrán A. ${ }^{1}$, José \\ Luis Rojas Arias ${ }^{1}$, Edgar Acuña Osorio ${ }^{1}$, Armicson Felipe Solano Montero. ${ }^{1}$ \\ 1 Unidad de Terapia, Cirugía Fetal y Fetoscopia, División de Medicina Materno Fetal, Departamento de Obstetricia y \\ Ginecología, Hospital de San José; Unidad de Medicina Materno Fetal, Fetoscopia y Cirugía Fetal, Clínica Colsubsidio \\ Orquídeas, Centro de Investigación en Salud Colsubsidio - CINCO; Departamento de Ginecología y Obstetricia, Funda- \\ ción Universitaria de Ciencias de la Salud - FUCS, Bogotá D.C., Colombia.
}

\section{RESUMEN}

Introducción: Los embarazos múltiples presentan riesgos de diferente naturaleza, frecuencia y severidad, debido a su comportamiento fisiopatológico particular según las características placentarias, impactando directamente en los resultados perinatales. Objetivo: Evaluar los resultados perinatales en embarazos gemelares teniendo en cuenta las características maternas, el resultado obstétrico, la morbilidad y mortalidad fetal y neonatal. Métodos: Estudio descriptivo de los resultados perinatales de embarazos gemelares en dos instituciones con presencia de grupo de medicina materno fetal, por medio de revisión de historias clínicas maternas y neonatales entre enero de 2009 y diciembre de 2012. Resultados: El promedio de edad materna fue 28,6 \pm 6 años, se incluyeron 438 gestantes y 885 gemelos de un total de 490 gestaciones múltiples, con nacimiento a las $34 \pm 4,1$ semanas de edad gestacional. Se presentó morbilidad neonatal severa en 127 casos (14,3\%), mortalidad neonatal temprana en 18 casos $(2 \%)$, admisión a la Unidad de Cuidado Intensivo Neonatal en 282 casos $(31,8 \%)$, la causa de ingreso más frecuente fueron las complicaciones respiratorias en 275 casos (31\%). Conclusiones: Nuestros hallazgos sugieren que el embarazo múltiple en nuestro medio, está asociado a complicaciones maternas, fetales, y neonatales, similar a lo reportado en la literatura, siendo mayores en los embarazos monocoriales biamnióticos. Se enfatiza en los embarazos múltiples, el cuidado prenatal, durante el nacimiento y postnatal, con el fin de mejorar los resultados tanto maternos como perinatales.

\section{PALABRAS CLAVES: Gemelos, embarazo múltiples, morbimortalidad perinatal, perinatología}

\section{SUMMARY}

Introduction: The risk of multiple pregnancies experience different nature, frequency, and severity, given its particular pathophysiological behavior as placental characteristics, directly impacting perinatal outcomes. Objective: To evaluate perinatal outcomes in twin pregnancies taking into account maternal characteristics, obstetric outcome, morbidity and fetal and neonatal mortality. Methods: Descriptive study of perinatal outcome of twin pregnancies in two institutions with presence of maternal fetal medicine group, through review of maternal and neonatal medical records between January 2009 and December 2012. Results: The mean maternal age was $28.6 \pm 6$ years, 438 pregnant women and 885 twins of a total of 490 multiple pregnancies were included, with birth at $34 \pm 4.1$ weeks gestational age. Severe neonatal morbidity in 127 cases $(14.3 \%)$, early neonatal mortality in 18 cases $(2 \%)$, admission to the Neonatal Intensive Care Unit in 282 cases $(31.8 \%)$, the cause was submitted admission were more frequent respiratory complications in 275 cases $(31 \%)$. Conclusions: Our findings suggest that multiple pregnancies in our environment, is associated with maternal, fetal, and neonatal complications, similar to that reported in the literature, being 
higher in monochorionic diamniotic pregnancies. So should emphasize prenatal care, during childbirth and postnatal care in multiple pregnancies, in order to improve both maternal and perinatal outcomes.

\section{KEY WORDS: Twins, multiple pregnancy, perinatal morbimortality, perinatology}

\section{INTRODUCCIÓN}

Los embarazos múltiples representan aproximadamente el $1 \%$ de los nacimientos, con aumento de sus tasas en las últimas décadas debido a la tendencia femenina de iniciar la gestación a edades tardías, y el uso de métodos de reproducción asistida (1-4). Las gestaciones dobles son los embarazos múltiples más frecuentes (95-98\%), sin embargo, representan tan solo el $3 \%$ del total de recién nacidos vivos $(2,4)$ y aún así, se relacionan con un $7,5-10 \%$ de mortalidad y alrededor del $21 \%$ de morbilidad perinatal $(3,5-8)$ con una alta tasa de compromiso materno y fetal, impactando social, cultural y económicamente en la población a pesar del aumento progresivo en la sobrevida global, por lo que los cuidados maternos, fetales y perinatales siguen siendo un reto en la actualidad (9).

Los embarazos múltiples se dividen según la cigocidad y corionicidad dependiendo del momento de la división celular, siendo la corionicidad el aspecto relevante para los resultados perinatales $(9,10)$. Se clasifican en monocigóticos y bicigóticos, teniendo 3 a 10 veces más complicaciones y riesgos los embarazos monocigóticos $(6,11,12)$. Si bien, el riesgo materno es similar, el riesgo fetal es sustancialmente mayor en embarazos monocoriales en comparación con los bicoriales $(13,14)$.

El embarazo múltiple expone a las madres y a su descendencia a riesgos extremadamente altos, especialmente en países en vía de desarrollo. No hay estudios suficientemente grandes sobre los resultados perinatales y maternos del embarazo gemelar en estas poblaciones (15).

El objetivo de este trabajo es evaluar los resultados perinatales en este tipo de embarazos en dos instituciones de referencia con presencia de grupo de alto riesgo obstétrico en un periodo de tres años, teniendo en cuenta el resultado obstétrico, morbilidad, mortalidad fetal y neonatal.

\section{PACIENTES Y MÉTODOS}

Se realizó un estudio observacional descriptivo en el Hospital de San José y la Clínica Colsubsidio Orquídeas entre 01 de enero de 2009 al 31 de diciembre de 2012. Se tomó el universo de la población incluyendo pacientes con embarazo gemelar, sin distinción de corionicidad y cigocidad, valoradas por la unidad de medicina materno fetal de cada institución, identificadas a través de las bases de datos del servicio de estadística, unidad de recién nacidos, certificados de recién nacidos vivos y de defunción, registros de patología, y de sala de partos. Se realizó revisión de los registros clínicos tanto maternos como de cada uno de los neonatos, extrayendo la información desde la primera valoración institucional hasta el egreso hospitalario, que se consideró de relevancia para el estudio. Se excluyeron pacientes con datos insuficientes.

Las variables usadas comprendieron datos sociodemográficos, antecedentes, datos de la gestación, vía del parto y resultados maternos, fetales y neonatales, enfocados en los resultados maternos (trastornos hipertensivos, síndrome HELLP, diabetes gestacional), resultados perinatales (malformaciones congénitas mayores, restricción del crecimiento intrauterino, síndrome de transfusión feto-fetal, secuencia TRAP), resultados neonatales definiendo morbilidad severa como la presencia de hemorragia intraventricular grado III o IV, enterocolitis necrotizante o requerimiento de ventilación mecánica y mortalidad neonatal temprana definida como la que se presenta en los primeros 7 días.

Los datos fueron almacenados en una base de datos de Microsoft $₫$ Excel $\circledast$ 2011, versión 14.0.0 (100825). El análisis de la información se realizó con el programa STATA versión $12 囚$. Las características sociodemográficas y clínicas se resumen con frecuencias absolutas y relativas, medidas de tendencia central y de dispersión, según el tipo de variable. El estudio fue aprobado por el Comité de ética de la facultad de Medicina de la Fundación Universitaria de Ciencias de la Salud (FUCS) y el Comité de ética de la Clínica Orquídeas Colsubsidio, considerándola investigación sin riesgo.

\section{RESULTADOS}

Se incluyeron 438 gestantes (429 gestaciones dobles y 9 triples), con un total de 885 gemelos (858 dobles y 27 triples). La prevalencia de los embarazos gemelares en el Hospital de San José fue $1,5 \%$ y en la Clínica Orquídeas de $1,7 \%$.

La edad materna promedio fue de 28,6 \pm 6 años; 319 pacientes eran multíparas (72,8\%), 119 primíparas $(27,1 \%), 1$ gestante tenía antecedente de embarazo gemelar $(0,1 \%)$. Los antecedentes médicos predominantes fueron las patologías endocrinológicas que se presentaron en 49 pacientes $(11,1 \%)$ siendo la más frecuente el hipotiroidismo; 18 pacientes eran fumadoras $(4,1 \%)$. Las características sociodemográficas y clínicas de las gestantes se describen en la Tabla I. 
Tabla I

CARACTERÍSTICAS SOCIODEMOGRÁFICASY MATERNAS EN 438 EMBARAZOS MÚLTIPLES

\begin{tabular}{|c|c|c|}
\hline \multicolumn{3}{|l|}{ Variable } \\
\hline Ocupación: & $\mathrm{n}$ & $\%$ \\
\hline Empleada & 223 & $(51,0)$ \\
\hline No empleada & 136 & $(31,0)$ \\
\hline No declara & 73 & $(17,0)$ \\
\hline Sin datos & 6 & $(1,4)$ \\
\hline Antecedentes médicos: & $\mathrm{n}$ & $\%$ \\
\hline Endocrinológicos & 49 & $(11,1)$ \\
\hline Infecciosos & 48 & $(11,0)$ \\
\hline Cardiovasculares & 15 & $(3,5)$ \\
\hline Malformaciones & 4 & $(1,0)$ \\
\hline Antecedentes tóxicos: & $\mathrm{n}$ & $\%$ \\
\hline Fumadora & 18 & $(4,1)$ \\
\hline Alcohol & 5 & $(1,1)$ \\
\hline Ambos & 9 & $(2,0)$ \\
\hline Antecedentes gineco-obstétricos: & $\mathrm{n}$ & $\%$ \\
\hline Primigestas & 119 & $(27,2)$ \\
\hline Multigestas & 319 & $(72,8)$ \\
\hline Antecedente de gemelar & 1 & $(0,1)$ \\
\hline Tipo de embarazo por ecografía: & $\mathrm{n}$ & $\%$ \\
\hline Bicorial Biamniótico & 236 & $(54,0)$ \\
\hline Monocorial Biamniótico & 192 & $(44,0)$ \\
\hline Monocorial Biamniótico, Monocorial Monoamniótico & 6 & $(1,4)$ \\
\hline Tricorial Triamniótico & 3 & $(0,7)$ \\
\hline Monocorial Monoamniótico & 1 & $(0,2)$ \\
\hline Multiplicidad: & $\mathrm{n}$ & $\%$ \\
\hline Doble & 429 & $(98,0)$ \\
\hline Triple & 9 & $(2,0)$ \\
\hline Patologías maternas: & $\mathrm{n}$ & $\%$ \\
\hline Hipertensión en el embarazo & 104 & $(23,8)$ \\
\hline Diabetes en el embarazo & 32 & $(7,3)$ \\
\hline Síndrome HELLP & 17 & $(3,9)$ \\
\hline Vía del parto: & $\mathrm{n}$ & $\%$ \\
\hline Cesárea & 416 & $(95,0)$ \\
\hline Vaginal & 22 & $(5,0)$ \\
\hline
\end{tabular}

Las gestaciones fueron clasificadas según multiplicidad, encontrándose 9 embarazos triples (2\%), y según corionicidad y amnionicidad con predominio de los bicoriales biamnióticos en 236 casos (53,8\%), seguido de monocorial biamniótico 198 casos $(45,2 \%)$, monocorial-monoamniótico 1 caso $(0,3 \%)$ y tricorial-triamniótico 3 casos $(0,6 \%) ; 328$ pacientes tuvieron parto pretérmino $(74,8 \%)$ y 110 $(25,1 \%)$ a término. La edad gestacional promedio de finalización del embarazo fue $34 \pm 4,1$ semanas. La vía del parto más frecuente fue la cesárea en 416 casos $(95 \%)$.

Las patologías maternas presentadas durante el embarazo fueron enfermedad hipertensiva en 104 pacientes $(23,8 \%)$, de las cuales $67(25,3 \%)$ correspondían a preeclampsia severa, $21(4,8 \%)$ a hipertensión gestacional, $7(1,6 \%)$ a hipertensión crónica, $7(1,6 \%)$ a preeclampsia no severa, $2(0,5 \%)$ a hipertensión crónica más preeclampsia sobreagregada. Se presentó síndrome HELLP en 17 casos $(3,9 \%)$. Diabetes gestacional en 20 casos $(4,6 \%)$, y diabetes pregestacional en 3 casos $(0,7 \%)$. Se documentó infección en 42 casos $(9,6 \%)$, siendo más frecuente la corioamnionitis en 11 casos $(2,5 \%)$, seguida por infección de vías urinarias en 8 casos $(1,9 \%)$. 
Las patologías fetales encontradas fueron restricción selectiva del crecimiento intrauterino en 58 gestaciones $(13,2 \%)$, síndrome de transfusión feto-fetal en 22 casos $(5,0 \%)$, secuencia TRAP en 4 casos $(0,9 \%)$, y muerte fetal en 34 gestaciones $(7,7 \%)$. Hubo muerte intrauterina de un feto en 16 embarazos $(3,6 \%)$ y muerte de ambos fetos en 17 casos $(3,8 \%)$, con promedio de edad gestacional de 25,1 $\pm 5,7$ semanas (Tabla II).

\section{Tabla II \\ CARACTERÍSTICAS DE LAS PATOLOGÍAS FETALES EN 885 FETOS DE EMBARAZOS MÚLTIPLES}

\begin{tabular}{lcc}
\hline Patología fetal & $\mathrm{n}$ & $\%$ \\
\hline $\begin{array}{l}\text { Restricción del crecimiento intrauterino } \\
\text { selectivo }\end{array}$ & 58 & $(13,2)$ \\
Síndrome de trasfusión feto-fetal & 22 & $(5,0)$ \\
Feto acárdico & 4 & $(0,9)$ \\
Muerte fetal: & 34 & $(7,7)$ \\
$\quad$ - Gestación doble & 32 & $(7,3)$ \\
$\quad$ - Gestación triple & 2 & $(0,4)$ \\
$\quad$ - Un solo feto & 16 & $(3,6)$ \\
$\quad$ - Ambos fetos & 17 & $(3,8)$ \\
$\quad$ - Tres fetos & 1 & $(0,2)$ \\
\hline
\end{tabular}

La discordancia de peso neonatal entre los gemelos ocurrió en un $20 \%$ de los embarazos múltiples, los resultados se expresaron de acuerdo a las semanas de gestación, entre $20-25$ semanas (7\%), 26-30 semanas (11,1\%), 31-35 semanas (1,37\%). No hubo discordancia de pesos en embarazos mayores de 35 semanas.

Las características neonatales se muestran en la Tabla III. Se presentó morbilidad neonatal severa en 127 casos $(14,3 \%)$, con predominio en el segundo neonato en 105 casos $(11,8 \%)$. Hubo mayor morbilidad en gestaciones dobles (123 casos; $13,8 \%$ ), y fue mayor en embarazos monocoriales biamnióticos (64 casos; 7,2\%). La mortalidad neonatal temprana se presentó en 18 casos (2\%), únicamente en gestaciones dobles, siendo más frecuente en embarazos monocoriales biamnióticos. De los neonatos evaluados se presentó mortalidad temprana en ambos neonatos en 13 casos $(1,5 \%)$ $y$ en un solo neonato en 5 casos $(0,5 \%)$.

Requirieron hospitalización en unidad de cuidado intensivo neonatal 282 casos $(31,8 \%)$, y las causas más frecuentes fueron: disfunción pulmonar en 275 casos (31\%), bajo peso en 55 casos $(6,2 \%)$ y sepsis en 44 casos $(4,9 \%)$. Las malformaciones mayores se presentaron en 22 casos $(2,5 \%)$, siendo las más frecuentes las múltiples asociaciones que se presentarón en 3 casos $(0,3 \%)$.

\section{DISCUSIÓN}

Los resultados perinatales de los embarazos gemelares continúan siendo un reto para la medicina materno fetal, por su asociación con una alta morbi-mortalidad materno y perinatal, siendo de vital importancia su identificación y manejo temprano (2,15-17).

En esta serie la prevalencia de gestaciones múltiples es de $1,5 \%$ en el Hospital de San José y $1,7 \%$ en la Clínica Colsubsidio Orquídeas en un periodo de tres años, siendo menor a las publicadas en las guías de práctica clínica del Instituto Mexicano del Seguro Social correspondientes al 3\% (2), pero similares a lo reportado por Vayssière y cols en Francia en 2008 de 1,5\% (18). Sin embargo, se conoce que la tasa de embarazo gemelar varía según la raza, la edad materna, y la situación geográfica, reportándose tasas entre 1,8 a 3,2\% (3).

La edad materna promedio de presentación del embarazo múltiple en nuestro estudio fue de 28 años, similar a lo reportado en otros estudios en donde el $87 \%$ se presentaron entre $18-35$ años de edad (15). La multiparidad también ha sido asociada como factor de riesgo para embarazo múltiple (15); en nuestro estudio el $72,8 \%$ de los embarazos gemelares se presentó en multíparas, encontrándose predominio de gestaciones dobles (98\%), similar a lo reportado por Ananth y Chauhan (95\%) (4).

El embarazo múltiple se ha asociado a altas tasas de parto pretérmino, que se han reportado hasta del $60 \%(4,15)$. En este estudio, encontramos tasas más altas $(74,8 \%)$, con edad gestacional promedio de finalización del embarazo de 34 semanas, acorde a lo reportado por Lee (19). Siendo la vía del parto más frecuente la cesárea (95\%), mucho mayor a lo reportado en la literatura (15), esto es debido a la aplicación del protocolo institucional en el que los embarazos múltiples se finalizan por cesárea. Sin embargo, se debe tener en cuenta que el parto vaginal se ha reportado en otras series hasta en el $57 \%$ (15).

El embarazo gemelar tiene mayor probabilidad de complicaciones maternas como trastornos hipertensivos, diabetes, anemia, parto pretérmino, hemorragia posparto y mortalidad materna $(1,15,20,21)$. En nuestro estudio, las complicaciones maternas más frecuentes fueron los trastornos hipertensivos del embarazo en sus diferentes presentaciones $(23,8 \%)$, similar a lo reportado por Young y Wylie con tasas hasta del $20 \%$ (21), seguida por diabetes gestacional, aunque estudios previos son contradictorios en cuanto al riesgo de diabetes gestacional en embarazos múltiples (21). 


\section{CARACTERÍSTICAS NEONATALES EN 885 NEONATOS}

\begin{tabular}{lcc}
\hline Peso neonatal (g) & $\bar{X}$ & $\pm \mathrm{DE}$ \\
\hline - Neonato 1 & 1997,5 & 618,7 \\
- Neonato 2 & 1969,5 & 627,7 \\
$\quad$ - Neonato 3 & 1298,4 & 705,5 \\
\hline Varias & $\mathrm{n}$ & $\%$ \\
\hline Discordancia de pesos fetales & 88 & 20 \\
Morbilidad neonatal severa & 127 & $(14,3)$ \\
Malformación fetal mayor & 22 & $(2,5)$ \\
Mortalidad neonatal temprana & $(2,0)$ \\
Un solo neonato & 18 & $(0,5)$ \\
Ambos neonatos & 5 & $(1,5)$ \\
Sin información & 13 & $(0,3)$ \\
\hline Diagnósticos clínicos neonatales en UCIN & 3 & $\%$ \\
\hline$\quad$ - Disfunción pulmonar & $\mathrm{n}$ & $(31,0)$ \\
$\quad$ - Bajo peso & 275 & $(6,2)$ \\
$\quad$ - Riesgo metabólico & 55 & $(2,2)$
\end{tabular}

El embarazo múltiple esta asociado con mayor frecuencia a resultados adversos y complicaciones perinatales, siendo la prematurez la principal causa $(1,5)$. Otras complicaciones son: anomalías fetales, bajo peso al nacer, parálisis cerebral, retraso en el desarrollo psicomotor, alteraciones en el comportamiento, así como enfermedad pulmonar y muerte entre el $2-7 \%$ de los casos $(5,16,22)$ y las dependientes de la corionicidad como síndrome de transfusión feto-fetal (STFF), restricción selectiva de crecimiento intrauterino y secuencia TRAP (1).

En nuestro estudio, se encontro restricción selectiva del crecimiento intrauterino en el $13,2 \%$ de los casos, similar a lo reportado por Valsky y cols con rangos entre $11,3-19 \%$, tomando como criterio diagnóstico la diferencia de pesos fetales mayor del $25 \%$ (23). El STFF se presentó en el 5\%, inferior a lo reportado en la literatura (10-15\%) (1). La secuencia TRAP se presentó en el $0,9 \%$ acorde con lo reportado por Chaloui y cols con presentación en el $1 \%$ de los embarazos monocoriales (13). Las malformaciones fetales mayores se presentaron en el $2,5 \%$ y la muerte fetal en el $7,7 \%$ similar a lo reportado en la literatura $(16,22)$.

Se presentó morbilidad neonatal severa en 127 casos $(14,3 \%)$, similar a lo reportado por Gezer y cols (8), con tasas de $15,4 \%$, a pesar de las altas tasas de prematurez que encontramos en nuestra población de $74,8 \%$ comparado con el $53,3 \%$ reportado en ese estudio. La morbilidad neonatal fue más frecuente en el segundo gemelo en 105 casos (12\%), acorde a lo descrito en la literatura, en donde históricamente se han reportado peores resultados en el segundo gemelo (6), el 14\% (123 casos) se presentó en embarazos dobles, siendo mayor en embarazos monocoriales biamnióticos en 64 casos $(7 \%)$, similar a lo reportado en estudios previos (8).

Los nacimientos por embarazos gemelares generan una alta proporción de partos pretérmino y aumento en la demanda de los servicios de cuidados intensivos neonatales $(4,15)$. En este estudio, el ingreso a la unidad de cuidado intensivo neonatal se requirió en 282 casos ( $31,8 \%)$, siendo la causa más frecuente el síndrome de dificultad respiratoria en el $31 \%$ de los casos, seguida de bajo peso al nacer y sepsis en 6,2 y $4,9 \%$ respectivamente, similar a lo reportado en estudios previos, en donde el ingreso a unidad de cuidado intensivo neonatal se requirió en el $28 \%$ de los casos y la principal causa de ingreso fue el síndrome de dificultad respiratoria (8). En nuestro estudio, se presentó mortalidad 
neonatal temprana en 18 casos (2\%), inferior a lo reportado en el estudio de Turquía, en donde se encontraron tasas de mortalidad neonatal temprana de $4,5 \%$ (8).

En nuestro conocimiento esta es la serie más grande de gestaciones múltiples en Latinoamérica, se estudiaron variables maternas, fetales y neonatales lo que permite establecer los resultados maternos y perinatales del embarazo múltiple. Se incluyeron todos los embarazos gemelares sin distinción de corionicidad y cigocidad, y es el primer estudio que establece la prevalencia de las complicaciones de estas gestaciones en nuestro país. Sin embargo, como limitaciones se presentaron dificultades por pérdida de información, dado por el carácter retrospectivo del estudio, pero consideramos que esto no invalida sus resultados.

\section{CONCLUSIONES}

Nuestros hallazgos sugieren que el embarazo múltiple en nuestro medio está asociado a complicaciones maternas, fetales y neonatales, similar a lo reportado en la literatura, siendo mayores en los embarazos monocoriales biamnióticos. Se debe enfatizar el cuidado prenatal, durante el nacimiento, y cuidado postnatal en los embarazos múltiples, con el fin de mejorar los resultados maternos y perinatales en nuestra población.

\section{REFERENCIAS}

1. Bricker L. Optimal antenatal care for twin and triplet pregnancy: the evidence base. Best Pract Res Clin Obstet Gynaecol 2014;28(2):305-17.

2. Instituto Mexicano del Seguro Social. Diagnóstico y manejo del embarazo múltiple. Evidencias y recomnedaciones. Catálogo maestro de guías de práctica clínica: IMSS-628-13. Mexico, D.F.: IMSS; 2013. p. 98.

3. Chauhan SP, Scardo JA, Hayes E, Abuhamad AZ, Berghella V. Twins: prevalence, problems, and preterm births. Am J Obstet Gynecol 2010;203(4):305-15.

4. Ananth CV, Chauhan SP. Epidemiology of twinning in developed countries. Semin Perinatol 2012;36(3):15661.

5. Black M, Bhattacharya S. Epidemiology of multiple pregnancy and the effect of assisted conception. Semin Fetal Neonatal Med 2010;15(6):306-12.

6. Ingram Cooke RW. Does neonatal and infant neurodevelopmental morbidity of multiples and singletons differ? Semin Fetal Neonatal Med 2010;15(6):362-6.

7. Hillman SC, Morris RK, Kilby MD. Single twin demise: consequence for survivors. Semin Fetal Neonatal Med 2010;15(6):319-26.
8. Gezer A, Rashidova M, Güralp O, Oçer F. Perinatal mortality and morbidity in twin pregnancies: the relation between chorionicity and gestational age at birth. Arch Gynecol Obstet 2012;285(2):353-60.

9. Wimalasundera RC. Selective reduction and termination of multiple pregnancies. Semin Fetal Neonatal Med 2010;15(6):327-35.

10. Stock S, Norman J. Preterm and term labour in multiple pregnancies. Semin Fetal Neonatal Med. 2010;15(6):336-41.

11. Gratacós E, Lewi L, Muñoz B, Acosta-Rojas R, Hernandez-Andrade $\mathrm{E}$, Martinez JM, et al. A classification system for selective intrauterine growth restriction in monochorionic pregnancies according to umbilical artery Doppler flow in the smaller twin. Ultrasound Obstet Gynecol 2007;30(1):28-34.

12. Sau A, Weber M, Shennan AH, Maxwell D. Antenatal detection of arteriovenous anastomoses in monochorionic twin pregnancy. Int J Gynaecol Obstet 2008;100(1):56-9.

13. Chalouhi GE, Stirnemann JJ, Salomon LJ, Essaoui M, Quibel T, Ville Y. Specific complications of monochorionic twin pregnancies: twin-twin transfusion syndrome and twin reversed arterial perfusion sequence. Semin Fetal Neonatal Med 2010;15(6):349-56.

14. Hack KE, Derks JB, Schaap AH, Lopriore E, Elias $\mathrm{SG}$, Arabin B, et al. Perinatal outcome of monoamniotic twin pregnancies. Obstet Gynecol 2009;113(2 Pt 1):353-60.

15. Vogel JP, Torloni MR, Seuc A, Betrán AP, Widmer M, Souza JP, et al. Maternal and perinatal outcomes of twin pregnancy in 23 low- and middle-income countries. PLoS One 2013;8(8):e70549.

16. Torres-Torres C, Perez-Borbon G, Benavides-Serralde JA, Guzman-Huerta ME, Hernandez-Andrade E. Prevalence and complications of monochorionic diamniotic twin pregnancy. Ginecol Obstet Mexico 2010;78(3):181-6.

17. D'Antonio F, Bhide A. Early pregnancy assessment in multiple pregnancies. Best Pract Res Clin Obstet Gynaecol 2014;28(2):201-14.

18. Vayssière $C$, Benoist $G$, Blondel $B$, Deruelle $P$, Favre R, Gallot D, et al. Twin pregnancies: guidelines for clinical practice from the French College of Gynaecologists and Obstetricians (CNGOF). Eur J Obstet Gynecol Reprod Biol 2011;156(1):12-7.

19. Lee YM. Delivery of twins. Semin Perinatol 2012;36(3):195-200.

20. Knox E, Martin W. Multiples clinic: a model for antenatal care. Semin Fetal Neonatal Med 2010;15(6):357-61.

21. Young BC, Wylie BJ. Effects of twin gestation on maternal morbidity. Semin Perinatol 2012;36(3):162-8.

22. Rand L, Eddleman KA, Stone J. Long-term outcomes in multiple gestations. Clin Perinatol 2005;32(2):495513, viii.

23. Valsky DV, Eixarch E, Martinez JM, Crispi F, Gratacós E. Selective intrauterine growth restriction in monochorionic twins: pathophysiology, diagnostic approach and management dilemmas. Semin Fetal Neonatal Med 2010;15(6):342-8. 